\title{
Enseñanza a distancia de la bibliotecología y estudios de la información
}

\author{
RAMIRO LAFUENTE LÓPEZ \\ ROBERTO GARDUÑo VERA \\ Centro Universitario de Investigaciones Bibliotecológicas \\ Universidad Nacional Autónoma de México, 04510 \\ México D.F., Tel: (525)623-03-55 y 525)623-03-45 \\ E-mail: lafuente@servidor.unam.mx \\ E-mail: garduno@servidor.unam.mx
}

\section{RESUMEN}

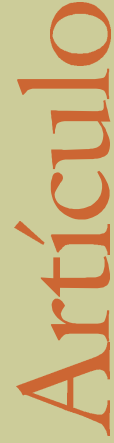

La creación de modelos educativos para la educación a distancia ha sido señalada como una alternativa para facilitarles a los individuos la oportunidad de acceder a programas educativos acordes con sus características ocupacionales. La reciente aparición de propuestas educativas a distancia ha puesto de manifiesto que los temas en torno a la construcción de modelos educativos en los que se incluya la convergencia de tecnologías de la información, requieren del análisis profundo de dos aspectos: la definición teórica del modelo y la forma de construir aplicaciones didácticas. A partir de estas consideraciones, en este artículo se abordan algunos aspectos relativos a la construcción de medios didácticos para la enseñanza a distancia desde la perspectiva del constructivismo, con la intención de analizar tópicos particulares de la enseñanza a distancia so bre temas vinculados a Bibliotecología y Estudios de la Información.

Trabajo recibido el

7 de diciembre

de 1999

*

Trabajo

aceptado el

27 de enero de 2000

Palabras clave: Educación a distancia, Modelos educativos, Enseñanza de la Bibliotecología

\section{DISTANCE TEACHING AND LEARNING OF LIBRARY SCIENCE \\ AND INFORMATION STUDIES \\ RAMIRO LAFUENTE-LÓPEZ \\ ROBERTO GARDUÑO-VERA}

\section{ABST RACT}

The creation of educational models for distance teaching and learning has been signaled as an alternative that could facilitate opportunities for individuals to study course-work that is in accord with their particular occupational characteristics. The recent emergence of distance learning proposals relying on the convergence of didactic models and information technology has demonstrated the need for in depth analysis regarding the theoretical definition of models and the mode of constructing didactic applications. In this light, this article employs a constructivist approach to analyze the creation of didactic material for use in distance teaching and learning of topics related to Library Science and Information Studies.

Key Words: Distance Education, Educational Models, Library Science Education 


\section{INTRODUCCIÓN}

F s perceptible latendencia encaminada a generar propuestas educativas en diferen-

L tes áreas del conocimiento, utilizando la modalidad de la educación a distancia con el fin de proporcionar alos individuos alternativas queles permitan acceder aprogramas educativos distintos a los presenciales. En este sentido se advierte, a partir de 1990, la propensión de diversas instituciones educativas a intensificar su oferta de enseñanza por medio de la educación a distancia, incremento que consideramos se puede atribuir a la tendencia social y política que apoya la idea de establecer una sociedad de lainformación y el conocimiento, sustentada en el uso intensivo de la tecnología de información y comunicación para apoyar los diversos procesos de la producción y organización social que requieren de nuevos conceptos educativos.

No obstante las bondades que puedan tener las tecnologías de información y comunicación, su uso requiere de una adecuada planeación y de la construcción de nuevos métodos de trabajo para organizar, administrar y certificar el proceso de enseñanza aprendizaje a distancia, lo cual requiere de:

* la inversión en infraestructura de tecnología de telecomunicaciones, quesirva desustetoa la creación y mantenimiento de repositorios de información, bibliotecas digitales, publicación electrónica, foros de comunicación para profesores y estudiantes, y procesos de control escolar;

* la creación de formas de gestión y el establecimiento de principios generales y normas apropiados para evaluar la calidad de los programas educativos, a partir de los niveles de los posibles estudiantes;

* la preparación de autores de material didáctico y la formación de tutores para operar este tipo de sistemas educativos.

Lainstauración de programas de educación a distancia en el campo delaBibliotecología y Estudios de la Información, requiere de una sólida comprensión sobre la naturaleza de esteámbito de estudio, y deuna adecuada sistematización de los conocimientos, hechos y fenómenos que lo conforman, así como el replanteamiento de las estrategias sobre los procesos de enseñanza-aprendizaje para adecuarlos tanto a la naturaleza de este tipo de enseñanza, como a deslindar cuáles de ellos podrían emplearse en el aprendizaje por medio de materiales de autoinstrucción y en el uso adecuado de las tecnologías de información y comunicación.

El diseño de programas de educación a distancia en Bibliotecologíay Estudios de la Información le plantea diversos retos a los autores de este tipo de programas. Por una parte hay que aplicar convenientemente las teorías y métodos característicos de este modelo, lo cual implicaun cambio deactitud en el maestro, quien debe concebir y practicar la enseñanza como un actor innovador y facilitador del aprendizaje. Por la otra, hay también que involucrarse en el diseño de materiales didácticos que respondan a métodos pedagógicos apropiados a los requerimientos de enseñar a aprender, y tener siempre en consideración las finalidades y las condiciones laborales, sociales y culturales de los estudiantes. Igualmente habrá que involucrarse en la 
utilización de tecnologías de educación a distancia, y en la creación de ambientes de comunicación por medios digitales que fortalezcan la generación de medios, y establecer comunidades de aprendizaje que permitan lafácil interacción entre la escuela, el tutor, el estudiante y los autores de materiales didácticos, dentro del marco del plan de estudios previsto.

En torno a lo anterior cabe señalar que las posibilidades ofrecidas por las tecnologías de lainformación han determinado que la generación de productos orientadosa la educación a distancia estén dejando de ser una actividad manual y dando origen a la producción de esquemas encaminados al uso de entornos tecnológicos de automatización, lo que puede facilitar el desarrollo de materiales de enseñanza que brinden múltiples opciones de interacción con el alumno, y le ayuden a conseguir un alto grado de conocimiento significativo.

Es difícil diseñar programas de educación a distancia en el ámbito bibliotecológico latinoamericano, dada la poca experiencia que tenemos en este ámbito, y porque hasta el momento estos programas han sido recibidos con recelo por parte de los especialistas en educación en general, y en educación bibliotecológica en particular. Las experiencias que existen se refieren a programas recientes, lo cual indica que hay que dejar madurar la aplicación de las teorías y metodologías propias del modelo de educación a distancia y fomentar el uso de tecnologías de información y comunicación. Se percibe que el nivel de licenciatura es el más favorecido por la enseñanza a distancia de la Bibliotecología y Estudios dela Información; en los posgrados, las especialidades, la educación continua y la capacitación, dicha modalidad casi no existe.

La educación a distancia en los ámbitos ya mencionados puede tener sus virtudes y limitaciones, y su desarrollo tendrá que ser muy selectivo mientras seacumulan conocimientos relativos al proceso mismo de enseñanza-aprendizaje acerca de los métodos y formas para configurar material didáctico sobre los tópicos específicos de estos campos de estudio. La preparación de materiales de autoinstrucción y la utilización de la modalidad de educación a distancia, pueden orientarse principalmente hacia la creación de recursos didácticos que actualicen los conocimientos y las especializaciones; posteriormente sería propicio hacer lo mismo con los posgrados. En este sentido, la educación a distancia es un elemento que puede coadyuvar a la construcción de un nuevo paradigma para la enseñanza de la Bibliotecología y los Estudios de la Información.

\section{CONSIDERACIONES TEÓRICAS CON RELACIÓN A LA EDUCACIÓN A DIST A NCIA}

La teoría so bre educación a distancia indica que cuando los modelos de enseñanza y estrategias de aprendizaje se utilizan en forma sistemática, puede valorarse con mayor claridad que el proceso educativo busca el desarrollo de las facultades intelectuales y morales de los individuos así como las formas y medios que respondan a las 
características de la propuesta educativa que se quiere instaurar, como lo menciona Cruz Rincón:

La educación es un proceso, individual y colectivo al servicio de la continuidad, la actualización y la renovación de una determinada cultura. El proceso educativo en cuanto dinámica cultural, debe propiciar la elaboración y el dominio en los grupos, de nuevos modelos de indagación de la realidad, de modelos valorativos y normativos para la acción y de formas de comunicación y expresión que afiancen la vinculación y cohesión del grupo o la comunidad. ${ }^{1}$

Lo anterior le es también inherente tanto a la educación presencial como a la educación abierta y a distancia. Sin embargo, el modelo de la educación presencial contempla la impartición del conocimiento en los entornos arquitectónicos de una institución escolar, y toma en cuenta los tiempos y edades predeterminados para el aprendizaje, los métodos de enseñanza y los contenidos de los planes y programas de estudio, cuya actualización se realiza por lo general sin considerar tiempos predeterminados; no obstante como indica Peña Borrero:

Aunque en un principio los estudiosos de la educación no formal insistieron demasiado en identificarla con aquella que se hace al margen de cualquier institución escolar, la experiencia ha demostrado que se puede hablar de proyectos de educación no formales adelantados por estas instituciones y que las modalidades así llamadas no formales tienden a institucionalizarse. Esto ha hecho que se amplíe mucho más el concepto, de manera que incluya otras tendencias des formalizantes en la educación. Si así se entiende, educación no-formal puede ser sinónimo de educación abierta. ${ }^{2}$

La discusión en torno a los asuntos antes mencionados ha generado dos posturas encontradas, una afirma que los conceptos de educación abierta y educación a distancia pueden ser considerados como sinónimos, y la otra señala que representan conceptos distintos. No obstante, es un hecho que ambos tienen connotaciones que pueden repercutir tanto en lo teórico como en la aplicación de las variables implicadas en dichos términos; en este sentido, conviene tener en consideración lo siguiente:

Universidad abierta designa una serie de cambios en el orden cualitativo, que no se restringen como en el de universidad a distancia a la cobertura espacial, un aspecto apenas dela apertura. Universidad abierta señala una finalidad, universidad a distancia se refiere más a un medio. Podría decirse quela primera se concentra en la intención (finalidad) y la segunda en la extensión (medios) ${ }^{3}$

Conviene señalar que la universidad abierta fue creada bajo una serie de preceptos filosóficos, mientras quela universidad a distancia se ha orientado al entorno de las estrategias, lo cual exige, entre otros aspectos, fundamentos teóricos que expliquen,

1 Jaime Cruz Rincón. Citado por Miguel Casas A rmegol En Univesidadsindases educaciónadistanciaen Améica Latina, 1986 p. 33.

2 Luis Alberto Peña Borrero. Planeacóndeprogramas deuniversidadabiataya distanaia: factarescúticos para la toma dedeisiones 1983, p. 9.

3 Ibidam 
identifiquen, determinen y posibiliten su desarrollo y aplicación, en este sentido, Casas Armegol, menciona lo siguiente:

La educación superior a distancia debe considerarse como una innovación tecnológica que se apoya en fundamentos teóricos, comunes y necesarios para comprender cualquier proceso educativo. Sin embargo, existe un conjunto de determinantes que proporcionan a esta modalidad educativa cierta singularidad que tipifica y justifica el empleo de determinadas estrategias y metodologías. ${ }^{4}$

El Reporte Willen de Suecia, indica que la educación a distancia consiste en: Una forma de educación donde la instrucción oral tiene un papel de subordinación y se concentra en periodos intensivos externos. Entre estos periodos el estudiante de manera individual, trabaja en casa pero tiene la posibilidad de consultar al maestro por teléfono o por carta. ${ }^{5}$

Por su parte, Peña Borrero, afirma que: La educación a distancia es una metodología de enseñanza que se caracteriza por la interposición de formas mediadoras entre docentes y estudiantes, para trascender las limitaciones de tiempo y espacio propios de la enseñanza directa o presencial y abrir así el ámbito de influencia de la educación. ${ }^{6}$

En consecuencia se puede señalar que la teoría sobre la educación a distancia está orientada a fundamentar una tecnología educativa basándose en términos filosóficos, teóricos, estructuras organizativas, metodologías de enseñanza aprendizaje, métodos para el diseño de materiales de autoinstrucción, procesos de comunicación, financiamiento, consecuencias sociales, culturales y políticas, etcétera. Ésta es la tendencia que ha sido aplicada en el modelo de la OpenUniversitydela GranBretaña, una de las experiencias en educación superior a distancia más exitosas y relevantes en el marco internacional que es reconocida por los estudiosos en educación como la experiencia pionera en la modalidad de la educación a distancia.

Posteriormente a la aparición de la OpenUnivesitydelaGranBretaña en las décadas de los setenta, ochenta y noventa surgieron bajo su influencia teórica las siguientes:

* Universidad Estatal a D istancia, en Costa Rica,

* Universidad Nacional de Educación a Distancia, en España,

* Universidad Nacional Abierta de Venezuela,

* Universidad de Athabasca, en Canadá,

* Universidad Para Todos los Hombres, en Israel, y la

* Syracuse University en Estados Unidos, entre otras.

Las corrientes prevalecientes en las universidades mencionadas en el párrafo anterior han pretendido darle un carácter humanístico y cultural a la educación a distancia declarándola como la clave para el crecimiento de un país, independientemente de

4 Miguel Casas Armegol, Universidad sin dases educacón a distancia en Améica Latina, 1986, p. 33.

5 G reville Rumble. On defining distance education. En [Antología del] Diplamada usos delas nuevas teendoǵas enla eduracóón México : UNAM, CISE, 1995. p. 67.

6 Luis Alberto Peña Borrero,. Planeación deprogramas deunivesidad abietaya distancia, p. 9. 
su nivel de desarrollo. Empero, diversos factores internos y externos han influido en los niveles de avance alcanzados por las universidades mencionadas, entre los cuales está la capacidad para incorporar las tecnologías de información y comunicación a los diversos procesos de enseñanza-aprendizaje.

No obstante, se puede señalar que desde el punto de vista teórico, los sistemas que se fundamentan en el modelo de la educación a distancia se caracterizan principalmente por:

* prestar una atención preponderante al desarrollo de materiales didácticos, de autoinstrucción;

* contemplar una población masiva predominantemente adulta de estudiantes;

* estar basados en una separación física entre docente y estudiantes, parcial 0 total, durante el proceso instruccional;

* requerir en forma esporádica contactos presenciales;

* necesitar medios particulares de comunicación entre estudiantesy tutores, y

* tender hacia el uso creciente de tecnologías de información y comunicación.

En este tipo de modelos la presencia física del maestro frente al estudiante se transforma, puesto quela interacción entre maestros y alumnos se establece a través de procedimientos y materiales didácticos de enseñanza aprendizaje dirigidos a fomentar en el estudiantela capacidad deaprender por sí mismo; este aspecto determinala necesidad de estudiar detalladamentela planificación del proceso educativo, así como la forma de aprender de los estudiantes.

El ambiente de comunicación de una educación a distancia debe prever quela enseñanza, en términos de espacio y tiempo, sea flexible, con el fin de facilitar el aprendizaje; por tanto debe tomar en cuenta que la actividad de aprendizaje por parte del estudiante puede darse en cualquier lugar y tiempo, en el momento que le sea propicio. Es fundamental tener presente esta circunstancia porque es una de las razones que obligan a que el diseño del material didáctico sea claro, preciso y cuente con todos los elementos para que el estudiante pueda desarrollar sus tareas de aprendizaje, puesto que es inevitable que éste podría, en ocasiones, no contar con la asesoría prevista en el plan de estudios.

\section{CONSIDERACIONES TEÓRICAS EN TORNO A LA CONSTRUCCIÓN DE MEDIOS DIDÁCTICOS PARA LA EN SEÑANZA A DISTANCIA}

La construcción de materiales didácticos para apoyar los procesos educativos de la educación a distancia es una de las tareas que le otorgan identidad propia a esta modalidad educativa debido a la relevancia que se le reconoce a estos materiales como parte medular del proceso de aprendizaje. Cabe advertir que el uso intensivo de medios de autoinstrucción en la educación a distancia tiende a condicionar los objetivos y los temas de los programas académicos, dado que la identificación y el tratamiento de los mensajes académicos requiere discriminar y estructurar dichos 
mensajes para que puedan registrarse con mayor facilidad, y tomar en cuenta las características particulares de los materiales didácticos que se pretenda utilizar.

Las tareas que exige el diseño de materiales de autoinstrucción indican que el estudiante es el sujeto principal del proceso de aprendizaje, por lo que es conveniente tomar en cuenta que es determinante:

[...] la formulación de objetivos, el análisis estructural de las tareas de aprendizaje, la selección de estrategias y medios adecuados para el logro de los objetivos, la combinación de estos de modo que constituyan un todo integrado, y el diseño de mecanismos que sirvan para recibir la información de retorno y evaluar permanentemente a los estudiantes. El empleo de modelos presupone además el conocimiento y aplicación de un conjunto de principios normativos tomados de las teorías del aprendizaje con los cuales se pretende hacer más científico y a lavez más eficiente el proceso de elaboración de los materiales.7

Una característica relevante respecto a los materiales que se emplean en la educación a distancia es su alto grado de estructuración didáctica debido a que su finalidad consiste en reforzar los aprendizajes; sin embargo, la búsqueda de un conocimiento más significativo tendría que tomar en consideración que los contenidos didácticos deben también ser objeto de un ordenamiento lógico, y permitir, con fundamento en bibliografías complementarias, la búsqueda de información adicional que hace el alumno sobre el tema que esté estudiando.

El alto grado de estructuración didáctica del material empleado en la educación a distancia se propone lograr una comunicación libre de ambigüedad tanto en la estructuración y secuenciación del material como en sus contenidos temáticos. Esto puede facilitarse recurriendo a modelos de diseño instruccional que indican cómo plantear objetivos de aprendizaje, cómo presentar el ordenamiento lógico de contenidos y cómo diseñar secuencias de aprendizaje que permitan que los estudiantes puedan corroborar sus avances y detectar las fallas de su aprendizaje. A este respecto, María Elena Chan, señala lo siguiente:

El proceso de enseñanza tiene como fin la formación de personas con capacidad para analizar, problematizar y actuar sobre su realidad y, por tanto, contribuir positiva y eficazmente en un proyecto social participativo. En este mismo tenor, al aprendizaje se le concibe como productivo, esto es, que el educando es capaz de crear ideas, desarrollary resolver problemas. ${ }^{8}$

Los hechos indican que los alumnos enrolados en programas a distancia por su condición de adultos exigen que los contenidos de aprendizaje les proporcionen no sólo la explicación de los fenómenos sino también su posible solución, aplicación y vinculación con los problemas que enfrentan cotidianamente en su centro de trabajo. En consecuencia deben preverse dos tipos de procesos educativos: el que se refiere a

7 Luis Alberto Peña Borrero. Planeacóndeprogramas deuniversidadabietaya distancia: factarescúticospera latoma dedaisiones Colombia; ICFES, Ministerio de Educación Nacional, 1983, p. 37.

8 Ma. Elena Chan Nuñez, y otros. "Guía para la elaboración del paquete de materiales didácticos orientados al aprendizaje independiente” En EstudioIndqpendiente México : ILCE, 1997. p. 260. 
lo informativo, que consiste en el cúmulo de información que necesita el estudiante para integrar el conocimiento, y el que tiene quever con lo formativo, a través del cual el sujeto aplica el conocimiento adquirido en la solución de problemas específicos

El diseño de materiales didácticos debe considerar diversas características pedagógicas, como las siguientes.

Epistemológica: la que atañe a la postura en torno a la construcción del conocimiento y que lleva a asumir una postura de articulación teórica para la formulación de la propuesta misma, y como principio central de la lógica misma que se recomienda para la elaboración de los materiales. Postura en la que se asume un sentido de totalidad relacional, en la quelos objetos de conocimiento no están predeterminados de antemano.

Teórica: se asumen dos vertientes principales en cuanto a la concepción de aprendizaje, la constructivista y la histórico-cultural. En ambas hay de fondo una postura epistemológica abierta y organicista. De ahí devienen conceptos como el de la problematización y la noción de integridad de las actividades y productos que se espera se construyan a través del desarrollo de las unidades didácticas. Se articula dentro de la perspectiva teórica el enfoque comunicacional sociocultural desde el cual se considera la cultura como el elemento fundamental de toda significación y se enfatiza el papel de los educandos, en tanto receptores activos que usan, significan y se apropian de los contenidos que son codificados por el elaborador del material de apoyo[... $]^{9}$

El papel que desempeña el autor de materiales didácticos es determinante en el proceso educativo que se genera con la educación a distancia, puesto que es él quien precisa y establece los medios idóneos para socializar los contenidos y comunicar la información que es relevante para el aprendizaje. En este sentido, el papel de la información es fungir como base paraintegrar el conocimiento que necesitan los estudiantes para construir estructuras de pensamiento que les permitan comprender los fenómenos y hechos que se estudian, y que los capaciten a utilizarlas como instrumento para identificary solucionar aquellos problemas que afecten su vida personal y profesional.

Con relación a lo expuesto en párrafos anteriores, la teoría indica la conveniencia de considerar, como básicos, al análisis de los objetivos generales y específicos de la propuesta educativa involucrada, considerando la vinculación temática que puedan tener las partes que estructuran el plan de estudios, con los requisitos de ingreso y egreso del programa y con la evaluación del conocimiento que el sujeto logró asimilar y debe evidenciar en términos materiales. Hay que corroborar los grados de aprendizaje logrados, comprobar si las habilidades programadas fueron alcanzadas, y evaluar la pertinencia de los contenidos y de las actividades de aprendizaje. La evaluación tiene sentido si se toma en cuenta el perfil que determina las habilidades que se espera lograr de los estudiantes con base en los contenidos, actividades, fuentes de información y productos que han elaborado.

9 Ibidem p. 261-262. 


\section{CONSTRUCTIVISMO Y APREN DIZAJE A DISTANCIA}

El diseño de materiales de autoinstrucción tiende a apoyarse en la concepción constructivista del aprendizaje, tomando en consideración que el proceso de construcción determina una actividad de reconstrucción y reestructuración; es decir, que el nuevo conocimiento se construye a partir del conocimiento adquirido con anterioridad por el alumno. Es el alumno quien construye su propio e individual conocimiento a través de su participación en actividades intencionales, planificadas y sistematizadas, orientadas a propiciar en éste, actividades mentales constructivas.

Sin embargo, la historia muestra que los cambios en la concepción del aprendizaje emergen de manera diferente en cada lugar en donde se adopta la educación a distancia; por lo tanto las teorías en las que se basa tal aprendizaje han sido influidas y orientadas por el ambiente social y el contexto económico y político del país que las asume. No obstante, es indispensable tener presente el fenómeno de globalización como un ingrediente importante si se quieren comprender y explicar algunas de las tendencias de la educación a distancia en estos finales del siglo XX.

El aprendizaje escolar y la intervención educativa basados en un plano constructivista, suponen diversas aproximaciones psicológicas aproblemas como los siguientes:

* El desarrollo psicológico del individuo, particularmente en el plano intelectual y en su intersección con los aprendizajes escolares.

* La identificación y atención a la diversidad de intereses, necesidades y motivaciones de los alumnos en relación con el proceso enseñanza-aprendizaje.

- El replanteamiento de los contenidos curriculares, orientados a que los sujetos aprendan a aprender sobre contenidos significativos.

- La búsqueda de alternativas novedosas para la selección, organización y distribución del conocimiento escolar, asociadas al diseño y promoción de estrategias de aprendizaje e instrucción cognoscitivas.

* Laimportancia dela promoción delainteracción entre el docentey sus alumnos, así como entre los alumnos mismos, a través del manejo del grupo mediante el empleo de estrategias de aprendizaje cooperativo.

* La revalorización del papel del docente, no sólo en sus funciones de transmisor del conocimiento, guía o facilitador del aprendizaje, sino más bien como mediador del mismo, resaltando el papel de la ayuda pedagógica que presta reguladamente al alumno. ${ }^{10}$

Con base en lo anterior, podemos señalar que las características de la enseñanzaaprendizaje que pueden darse a través del proceso educativo basado en el constructivismo, se relacionan con:

* el modo en que se adquiere el conocimiento, el cual puede darse a través de la percepción y el descubrimiento y,

10 Frida Díaz Barriga,. "El aprendizaje significativo desde una perspectiva constructivista." ED UCAR. 1993: p.24. Citada por G uillermo G arcía O lvera. En Propursta paradaborarprogramasdeac tualizacónadistanciaparaegesadosdeesaudasmexicanadebiblideedoǵa(tesis de Maestría en proceso). 
* la forma en que el conocimiento es incorporado en la estructura cognoscitiva del estudiante, que puede ser por repetición y significativo.

La teoría menciona que la estructura cognoscitiva del estudiante tiene una serie de antecedentes y conocimientos previos, un vocabulario y un marco de referencia personal, todo lo cual indica aspectos relacionados con su madurez intelectual. Por tanto estas variables son determinantes al diseñar y elaborar material didáctico para la educación a distancia.

\section{TECNOLOGÍAS DE INFORMACIÓN, COMUNICACIÓN Y EDUCACIÓN A DISTANCIA}

La aparición de programas de educación a distancia a partir de la década de los noventa ha puesto de manifiesto que los marcos conceptuales utilizados con anterioridad deben ser modificados para incorporar el uso y la enseñanza de tecnologías de información y comunicación en los diversos procesos educativos. El rápido desarrollo de las tecnologías de telecomunicación así como la incorporación de multimedios a los procesos educativos, la aparición del teletrabajo, el telemercado, y la educación en línea, son aspectos que seguramente estarán presentes con mayor énfasis en la educación del nuevo siglo.

Se advierte que a la telemática educativa le es inherente la educación en línea, cuyo propósito principal es la creación de aulas virtuales o universidades virtuales en las que los alumnos y tutores se comunican a distancia a través de multimedios que combinan texto, datos, sonido e imágenes, y que utilizan la automatización como soporte. En consecuencia, la telemática, la informáticay el multimedio educativo requieren de la automatización para hacer factible la transmisión de contenidos y la comunicación entre alumno y tutor.

Es evidente que las tecnologías más recientes ofrecen diversas posibilidades de comunicación con el alumno, controles académicos versátiles, y diseño de materiales didácticos basados en estructuras de hipertexto e hipermedio. También es claro que después de los noventa se acentúa el uso de la videoconferencia, el correo electrónico, la biblioteca digital y la publicación digital, y seadvierte la generación de servicios de diseminación selectiva de la información bibliográfica vía las telecomunicaciones y la transferencia de documentos digitales.

Así, las tecnologías de información y comunicación ofrecen diversas posibilidades para el diseño de modelos educativos y de materiales didácticos con fines de docencia e investigación. Esto pone de manifiesto que la incorporación de tecnologías de información y comunicación en la educación a distancia requiere de una reordenación teórica dirigida principalmente al desarrollo de conceptos, definición de estructuras y normas para construir materiales didácticos que garanticen el aprendizaje y la adquisición de conocimiento significativo. 
Con el uso de tecnologías de información y comunicación, un rubro importante que atañe a la tecnología educativa es una pedagogía que sea "autoinstructiva, interactiva, personalizadora y que a su vez, incida sobre el aprendizaje, los medios y la metacognición." 11 La construcción de modelos integrales en línea deben tomar en cuenta estos propósitos y desarrollar bases de conocimiento flexibles y que muestren el uso pertinente de la teoría que subyace en la educación a distancia, principalmente en los siguientes asuntos:

* Construcción de multimedios que le faciliten al alumno la reconstrucción de sus conocimientos previos con el fin de que genere nuevas estructuras de conocimiento.

* D iseño de ambientes de aprendizaje que contemple el conjunto de actividades, experiencias y recursos de apoyo y respalde la reconstrucción de conocimientos.

* Infraestructuras de comunicación remota que faciliten lainteracción no presencial entre tutores y alumnos a través de actividades como discusiones, intercambio de ensayos, referencias bibliográficas, materiales de lectura y visita de sitios web relacionados con temáticas relevantes a los cursos.

* Construcción de foros de discusión virtuales que faciliten la comunicación asíncrona, rica en reflexiones, entre los alumnos, independientemente del área geográfica en la que se encuentren.

Un tema que se destaca en la educación a distancia es el diseño de seminarios virtuales, considerados como un intercambio de información relevante que propicia la reflexión sobre un tópico especifico. Aquíla comunicación se establece entre personas situadas en distintos lugares y es mediada por sistemas de telecomunicación, programas y reglas que ordenan la información que se quiere transferir a sujetos que acceden a ella en distintos momentos. El seminario virtual puede ser utilizado en actividades de larga duración, y es conveniente que su diseño contemple aspectos como los siguientes:

* que incluya bases de conocimientos elaborados con fundamento en teorías apropiadas a los contenidos informativos multimedia.

* que desarrolle conceptos y finalidades que orienten tanto la inclusión de información como las pláticas en vivo, ya sea por dhato audio digitalmente.

* que muestre de forma explícita los objetivos iniciales y terminales del seminario.

* que determine el perfil de los participantes y considere sus capacidades de acceder a la infraestructura tecnológica.

11 Antonio J. Colom C., en su trabajo titulado "Pedagogía tecnológica para la educación a distancia", se propone demostrar por qué la pedagogía que él llama tecnológica es el constructo teórico más pertinente y el que mejor se adapta al desarrollo de la EAD. 
* que incluya enlaces a recursos informativos disponibles en Internet (páginas web y bibliotecas digitales de contenidos temáticos relevantes para el tema en cuestión).

No obstantela existencia de diversas propuestas didácticas en línea, se adviertela necesidad de darle una mayor atención a su estudio sistemático, lo que permitiría confrontar los resultados de su uso y desarrollar parámetros acerca de su diseño, construcción y aplicación a la enseñanza de la Bibliotecología y los Estudios de Información. En consecuencia, el estudio de estas cuestiones debería ser un compromiso obligado para la investigación bibliotecológica.

\section{En SEÑANZA A DISTANCIA DE LA BIBLIOTECOLOGÍA}

Podemos asumir que los tópicos anotados con anterioridad le son inherentes a cualquier propuesta educativa referida a la enseñanza de la bibliotecología, sin embargo, es necesario analizar su aplicación a la luz de las características de la disciplina y de su ejercicio. En este sentido, es indispensable contemplar que la enseñanza de la Bibliotecología debe cumplir con la intención básica de que el estudiante adquiera conocimientos sobre el desarrollo de la disciplina, sus supuestos y el valor que tiene. Tendría que comprender que los fenómenos bibliotecológicos se presentan en entornos sociales específicos y que su evolución ha generado instituciones sociales denominadas bibliotecas, que organizan preservan y difunden la cultura, y cuyo conocimiento ha dado lugar a una taxonomía que las clasifica en tipos y categorías: Bibliotecas Públicas, Bibliotecas Nacionales, Bibliotecas Universitarias, Bibliotecas Especializadas, Centros de documentación, Centros de información, etcétera.

La enseñanza de la Bibliotecología y los Estudios de la información debe proponerse la obtención de una adecuada formación teórica que le permita al estudiante comprender los fenómenos relativos a este ámbito del conocimiento, y la adquisición de las habilidades necesarias para seguirlo estudiando. Sin embargo, para desarrollar una apropiada actividad profesional es indispensable que la formación teórica se equilibre con la enseñanza de destrezas y habilidades, y que se apliquen los conocimientos adquiridos en el diseño y la operación de los servicios de información documental de todo tipo. La enseñanza de destrezas y habilidades es una cuestión compleja porque requiere sincronizar diversos factores y evitar caer en la enseñanza de procedimientos memorísticos, repetitivos y mecánicos, desprovistos de toda cualificación. Es fundamental enseñar no sólo a hacer "algo", sino el ¿cómo y él para qué hacerlo? lo cual implica esencialmente un dominio del conocimiento del campo de la disciplina, y una sólida formación en el diseño y la construcción de guías didácticas para el cumplimiento de objetivos específicos.

En otros términos, la enseñanza a distancia de la Bibliotecologíay los Estudios de la Información requiere de recursos didácticos que auxilien a los estudiantes en el aprendizaje de los principios y teorías orientados a la comprensión de la naturaleza 
de los fenómenos inherentes a este campo de estudio. La intención debe ser que el estudiante se apropie de los conocimientos suficientes para identificar y explicar tales fenómenos, pero ha de buscarse en todo momento que sea capaz de vincular estos conocimientos con su aplicabilidad pragmática.

No se trata, por ejemplo, de que aprenda los fundamentos de la organización documental, sino que los aprenda con la intención de aplicarlos en el manejo de reglas, formatos bibliográficos, formatos digitales, bases de datos, catálogos en línea, sistemas metadatos, servicios de información, etcétera. El objetivo será que adquiera las habilidades suficientes y pueda aplicarlas a la solución de casos específicos relacionados con la organización de documentos que habrá de encontrar en el ejercicio de la profesión.

Contemplar la enseñanza delabibliotecologíay los estudios delainformación en el sentido mencionado, implicalaformación de un alumno a partir de la selección y el ordenamiento delos conocimientos disponibles con lafinalidad de sustentar la enseñanza en aquéllos que le faciliten una formación que le permita ejercer su profesión y seguirse actualizando, dada la naturaleza de un mercado de trabajo sujeto a constantes cambios. Una enseñanza de esta naturaleza requiere de recursos didácticos que respondan a la idea de que el proceso de enseñanza-aprendizaje debe estructurarse conformeaun orden de prioridades lógicas. Se trata de darleal estudiantelos conocimientos que necesita para comprender un fenómeno, pero también aquellos elementos que lo induzcan a reflexionar y a identificar un problema y la forma de solucionarlo. En consecuencia, es esencial que el alumno de un sistema de educación a distancia cuente con material de autoinstrucción que le proporcione fundamentos para comprendery explicar las relaciones que existen entre los fenómenos que debe discernir.

La enseñanza en el nivel de licenciatura tendría que dotar al alumno primordialmente de los conocimientos básicos de la disciplina para poder desempeñarse en el ejercicio dela profesión con el fin de socializar su conocimiento y las habilidades adquiridas durante su formación. Por su parte, la enseñanza de postgrado ha de tender a profundizar el estudio de la Bibliotecología y los Estudios de la información para generar en el estudiante habilidades docentes y de investigación.

En este sentido, la enseñanza de la disciplina requiere de marcos referenciales que faciliten entender su concepción con el propósito de identificar los fenómenos y problemas que deben ser estudiados. "[...] si consideramos a la Bibliotecología como la ciencia que estudia: el registro y flujo del conocimiento y de la información; así como la circulación social de los medios que la contienen para hacer posible su uso y organización." 12 Entonces la consecuencia será que el estudiante debe: estudiar epistemología de la Bibliotecología y de los Estudios de la Información así como las diversas formas de producción y circulación de documentos como vehículos de difusión para la socialización del conocimiento y de la información; entender

12 Lafuente López, Ramiro y Estela Morales. Reflexiones en torno a la enseñanza de la bibliotecología. En InvestigaciónBiblideedógica, 6(12) p.25, 1992. 
y explicar las diferentes formas de producción, así como el papel que juegan autores y editores, y los derechos que se involucran en este tipo de actividades; conocer los flujos de la información en diversos entornos geográficos y en diversas sociedades; comprender los fundamentos de la selección de documentos atendiendo a los requerimientos de distintas comunidades; dominar los métodos del análisis de documentos para objetivar su representación con fines de organización y recuperación manual y/ o automatizada; identificar las tendencias y utilidad de las tecnologías de información y comunicación para el manejo de documentos y las características de las comunidades de usuarios de la información, etcétera.

Incidir en una formación que contemple entre otros objetos de estudio, los señalados con anterioridad, requiere:

[...]agrupar los fenómenos en áreas de conocimiento que permitan aglutinar con propósitos didácticos fenómenos afines o que sean necesarios como antecedente conceptual para la comprensión o explicación de otros. ${ }^{13}$

Sin embargo, la agrupación de fenómenos de estudio implica reconocer que no existe hasta el momento una clasificación de los conocimientos de la disciplina que esté completamente validaday sea de uso general. Por otra parte la literatura identifica que existen varias propuestas de clasificación, algunas de las cuales han servido como fundamento para continuar con el estudio del cuerpo de conocimientos que debe tener la Bibliotecología como disciplina. No obstante aún queda mucho por hacer, pues:

[...] la organización de los conocimientos bibliotecológicos que tiene una mayor aceptación aún corresponde a los esquemas clásicos de la organización técnica de la biblioteca, a saber: Servicios, Catalogación, Clasificación, y Usuarios. ${ }^{14}$

Para replantear la enseñanza de la Bibliotecología y los Estudios de la información y adecuarla a los nuevos fenómenos que presenta la organización y difusión del conocimiento y la información, es necesario validar, jerarquizar e interrelacionar el campo de estudio y los conocimientos existentes sobre él, a fin de lograr una adecuada organización didáctica que establezca las pautas necesarias que puedan transmitirle al estudiante una visión integradora del conocimiento.

Lo anterior implica también resolver problemas relativos al orden en que deberán agruparse los diversos fenómenos, los conceptos, las teorías y las técnicas por lo que toca a las prioridades lógicas de enseñanza, las cuales se basan en la identificación de conceptos primarios y el aumento gradual de la complejidad del manejo conceptual. La intención es que el estudiante sea capaz de manejar e interrelacionar los conceptos relativos a la explicación o comprensión de los fenómenos motivo de la enseñanza.

13 Ibidem p. 25.

14 Ibidem p. 26. 
Abordar la enseñanza adistancia en el ámbito de la Bibliotecologíay los Estudios de la Información también implica considerar que el fenómeno de globalización ha penetrado en las sociedades con laintención de eliminar barreras de comunicación y facilitar los flujos de la información a través de la tecnología digital, la informática y las telecomunicaciones. En consecuencia, el estudio de la globalización implica, asu vez, el conocimiento y manejo de habilidades referidas a las particularidades tecnológicas de dichas herramientas, sobre todo si consideramos que están produciendo un nuevo tipo de repositorios documentales de carácter digital a partir de los cuales habrá que implementar diversos tipos de servicios.

La globalización y las tecnologías de la información son inherentes al campo de estudio de la Bibliotecología y Estudios de la Información, debido a que ambos fenómenos han puesto de manifiesto la necesidad de contar con nuevos perfiles profesionales para el desarrollo de actividades en el terreno de los documentos digitales y su transmisión vía redes de telecomunicación. A este respecto baste señalar que la introducción del documento digital y las telecomunicaciones vía redes, han obligado a replantear la selección y adquisición de documentos impresos y digitales, así como su sistematización, su transferencia, la prestación de servicios de información y la atención al usuario final de los servicios bibliotecarios.

En la actualidad un hecho que puede resultar simplista es la existencia de usuarios que consideran que labiblioteca moderna debe tener por lo menos sus catálogos automatizados, prestar servicios de diseminación selectiva de la información y tener acceso a Internet. En términos de enseñanza-aprendizaje, lo anterior naturalmente no tiene nada de simpley más bien lleva al planteamiento de fenómenos de estudio y de enseñanza-aprendizaje, puesto que implica incidir en la formación de un profesional que identifique, detecte organice, cree, difunday comprenda la naturaleza del manejo de documentos a través de las tecnologías mencionadas. Se busca, que entienda cómo influyen estas tecnologías en áreas estratégicas para el desarrollo local, nacional y regional de determinado país. De persistir la tendencia hacia la globalización, requeriríamos recursos humanos que pudieran sistematizar, seleccionar y transferir documentos digitales, aspectos que rebasan la sola adquisición de habilidades en el manejo de tecnologías y que hacen pensar más en la necesidad de aprender diversas opciones para manejar la información documental de manera creativay benéfica para la comunidad.

La sociedad actual creaformas inéditas de generación y uso de documentos tanto impresos como digitales, y ha producido diferencias en cuanto a la capacidad de las personas no sólo para acceder al documento, sino incluso para reconocerlo y saber usarlo, lo cual ha originado diversos tipos de marginación cultural tanto nacionales como locales. Estas nuevas circunstancias sociales requieren de un profesionista que tenga la capacidad, de identificar y caracterizar escenarios culturales, sociales y económicos en los que sea factible aplicar y utilizar las tecnologías de información y realizar una adecuada gestión de la documentación que se produce, así como diseñar 
colecciones de documentos y sistemas de información acordes con las finalidades informativas de una determinada comunidad.

Al respecto, con el fin de construir un posible perfil del egresado que requiere nuestro tiempo Sánchez y Pérez proponen que se den cuatro "saltos cualitativos" desde el punto de vista epistemológico:

- El primer salto cualitativo, va del documento a la información.

* El segundo, del énfasis de los procesos de acceso y transmisión de la información a un nuevo énfasis en los procesos comunicacionales al servicio de los usuarios finales.

- El tercero, va de los servicios diseñados desde una perspectiva localista a una perspectiva cooperativista de servicios en redes.

- El cuarto, apunta al dominio y conocimiento de los procesos cognitivos, comunicacionales y linguísticos generados por el cambio de soporte de los documentos. 15

Así, se esperaría que los egresados de programas en Bibliotecología y estudios de la información a distancia, desarrollaran habilidades para:

- Agregar valor: esto es detectar, analizar, sintetizar, interpretar y evaluar información y conocimiento. D esarrollar capacidad para discernir problemas relevantes, desarrollar un pensamiento crítico en relación a la información, su acceso, ordenamiento e investigación, desde una perspectiva inter y multidisciplinaria.

— Técnicas: que garanticen un uso eficiente y eficaz de las tecnologías que hacen posible la comunicación inter-redes, y la organización, procesamiento, acceso, transmisión y distribución de la información.

— Gerenciales: que partan de principios de análisis, planificación, instrumentación y control articulados a programas y políticas destinadas a alcanzar objetivos y metas específicas. Estudio y manejo de los paradigmas organizacionales vinculados al desarrollo de las tecnologías de información y comunicación electrónica; funcionalidad del trabajo en ambiente de redes, organización del trabajo bajo sistemas integrados. Liderazgo en contextos organizacionales cambiantes.

* Sociales: capacidad para la comprensión del entorno macro-social, de las exigencias de la realidad nacional en materia de información y diseño de políticas de información y tecnología destinadas a actuar sobre esa realidad. ${ }^{16}$

En los últimos años, diversas escuelas de Bibliotecología de A mérica Latina se han dado a la tarea de revisar sus planes y programas de estudio a raíz de la aparición de nuevos fenómenos que requieren ser metabolizados. México, ha realizado un intenso trabajo de revisión y adecuación de los planes y programas de estudio de las Licenciaturas en Bibliotecología del país y dela Maestría en Bibliotecología de laUNAM, modificaciones que ya están vigentes este año; asimismo el D octorado en Bibliotecología y Estudios de la Información, también de la UNAM, se inicia a partir del 2000.

15 Saadia Sánchez V. y Estrella Pérez M. "Reflexiones sobre la formación de recursos humanos de cuarto nivel en el área de las ciencias de la información". En INFOLAC. 9(4),1996.

16 Ibidem p.7. 
El planteamiento actual de la Licenciatura en Bibliotecología de la UNAM se estructura en seis áreas, a saber: Fundamentos disciplinarios, Control Bibliográfico, Gestión, Tecnología, Investigación y docencia, Recursos y servicios. Esto se complementa con nueve asignaturas optativas cuyas temáticas giran en torno a las seis áreas mencionadas.

La propuesta más reciente de la Maestría en Bibliotecología e Información de la UNAM, se agrupa en cinco áreas temáticas: Información, conocimiento y sociedad, O rganización de la información documental, Tecnologías de la información, Sistemas y servicios bibliotecarios y de información y, Usuarios de la información.

Por su parte, la propuesta del Centro Universitario de Investigaciones Bibliotecológicas (CUIB) de la UNAM, incluye cinco áreas de investigación: Fundamentos de las Ciencias Bibliotecológicas y de Información; Información y sociedad; Sistemas de información; Análisis y sistematización de la información documental, y Tecnologías de la información.

Con base en lo anterior, se puede apreciar que existe interés por parte de los administradores de escuelas en incidir en la revisión de planes y programas de estudio con el fin de incorporar a la curricula los nuevos conocimientos sobre las circunstancias actuales. A simismo la investigación se ocupa de identificar y estudiar los fenómenos involucrados en el uso de tecnologías de información y comunicación.

\section{CONSTRUCCIÓN DE MEDIOS DIDÁCTICOS PARA LA EN SEÑANZA A DISTANCIA DE LA BIBLIOTECOLOGÍA}

Un indicador relevante para la construcción de materiales didácticos es que los alumnos que ingresan a las escuelas de Bibliotecología y E studios dela Información tienen como objetivo principal el ejercicio de la profesión, pues por lo general han laborado en diversas unidades de información dedicadas a la prestación de servicios documentales. Para ellos la consultoría como una expresión del ejercicio de la profesión es un fenómeno relativamente nuevo y escaso en el medio, y la investigación científica, tecnológica o académica es una circunstancia más bien excepcional. Esta situación determina que la mayoría de alumnos potenciales de un programa de educación a distancia, estén inmersos en la solución de problemas relacionados con el ejercicio de la profesión einvolucrados en el funcionamiento adecuado de las unidades de información donde laboran.

El ejercicio profesional en el terreno propio del manejo de servicios de información do cumental, implica una actitud de carácter pragmático cuya finalidad esencial es hacer funcionar los procesos de organización y búsqueda documental de tal manera que el profesional satisfaga los requerimientos inmediatos de la unidad de información para la cual trabaja. Sin embargo, la mayor fuente de problemas para el ejercicio 
profesional no se genera en esas unidades, más bien se debe a los cambios surgidos en el ambiente social donde se encuentra o trata de incidir la unidad de información.

Naturalmente la comprensión y explicación de los fenómenos relacionados con la organización y manejo de información documental paralograrun uso más efectivo de ella, no se contrapone al necesario pragmatismo al que obliga el ejercicio profesional en estos terrenos; por el contrario, lainterrelación constante entre ambos aspectos debería propiciar una mayor riqueza en el desarrollo de conocimientos so bre estas cuestiones, sin embargo, no es éste un asunto de fácil solución debido a que la multiplicidad de fenómenos que convergen lo convierten en un asunto muy complejo.

El diseño de materiales para la educación a distancia debe estar enmarcado por un contexto. Por ejemplo, un material para enseñar catalogación puede responder a las necesidades curriculares de un sistema escolarizado a distancia, o formar parte de las estrategias de capacitación de alguna institución. En este sentido el autor del material didáctico es quien determina el propósito de su trabajo, pero lo efectúa con relación al cumplimiento de fines que trascienden sus intereses personales inmediatos, y que se encuentran en relación con las finalidades perseguidas por una institución 0 un círculo social.

Por ejemplo, los procesos de enseñanza-aprendizaje sobre conocimientos bibliotecológicos orientados a la organización documental, requieren de la presencia simultánea de la explicación de un fenómeno y la demostración de la aplicabilidad del conocimiento a lasolución de problemas específicos. El objetivo sería mostrar el tipo de procesos y procedimientos que es factible obtener, como podría ser la organización y generación de flujos de información bibliográfica; el diseño de bases de datos, y el diseño de sistemas de metadatos. Lo anterior requiere necesariamente que el alumno tenga una formación conceptual que le facilite la comprensión de la diversidad defenómenos presentes en la sistematización documental, y de las repercusiones que éstos puedan tener en los servicios de información.

Los fenómenos presentes en el proceso de control bibliográfico y manejo de fuentes de información para resolver problemas específicos de localización de documentos, son aspectos relevantes para la organización documental; en consecuencia, los materiales didácticos para enseñar organización do cumental deben hacer explícitos los objetivos que se persiguen, y ser éstos expresados en términos que le permitan a quien los utiliza, mensurar la adquisición de información y la comprensión delos procesos que se trata de explicar. Lo anterior supone una secuencialógica en la presentación de los contenidos instructivos de los materiales; en este sentido, se debe tener presente que el contexto es indispensable para comprender un determinado proceso , pero resultaigualmente importante mostrar la forma de hacer operativo un proceso bajo diversas condiciones de aplicabilidad.

Por lo anterior, la presentación y organización de materiales didácticos involucrala especificación del tipo de información que debe incluirse, la secuencia en que debe estructurarsey laforma en que deben interrelacionarse el texto y el contenido gráfico. Es necesario que se incluyan descripciones y detalles específicos acerca de los procesos e 
instrucciones desmenuzadas so bre la aplicación de técnicas y procedimientos, y que todo esté preferentemente acompañado de ejemplos prácticos de fácil aplicación.

La metodología parala elaboración de materiales didácticos indica que si se pretende mostrar la funcionalidad de un determinado tipo de técnica, es factible estructurarlo de acuerdo con una amplia gama de posibilidades, que pueden ir desde la simple presentación o descripción específica hasta una elaborada estructura destinada a facilitar el uso de una determinada técnica. La estructura y el contenido del material predeterminan en cierta medida su utilidad al establecer un contexto en cuanto a su uso.

A simismo es necesario someter al material didáctico a procesos de validación y verificación. La validación involucra un proceso de revisión por parte de personas expertas en el tema del material, con objeto de evitar errores técnicos o un manejo inadecuado del lenguaje, los conceptos o los procedimientos. La verificación implica comprobar si efectivamente el uso del material responde o no a las finalidades previstas, y si las instrucciones son pertinentes y claras. Laverificación debe realizarse con personas cuya competencia se considere similar a la de quien vaya a usar el material didáctico.

\section{ELEMENTOS PARA EL DISEÑO DE UN MOdELO DE EDUCACIÓN A DISTANCIA}

En términos generales la enseñanza de la Bibliotecología y de Estudios de la Información es una actividad a menudo considerada como algo simple porque existe poca comprensión acerca de los componentes que se ponen en juego para que ésta funcione. No obstante, su enseñanza involucra un comportamiento complejo en el que interactúan diversos elementos. El propósito del presente modelo es identificar los elementos que debe considerar la creación de materiales didácticos, y las relaciones que es necesario establecer entre diversos elementos para inducir procesos de aprendizaje. El modelo gira en torno de la creación de materiales didácticos dado que en la modalidad de educación a distancia éstos constituyen el eje del proceso de enseñanza-aprendizaje.

El modelo reconoce cuatro componentes principales:

* diseño de material didáctico,

* determinación de elementos para conduccir la asesoría,

* procedimientos de enseñanza, y

* proceso de evaluación.

A continuación se despliega un cuadro general que agrupa los diversos aspectos que forman parte de cada uno de los elementos mencionados. En párrafos subsecuentes se detalla cada uno de estos aspectos. 


\section{PROCESO PARA EL DESARROLLO DE MATERIALES DIDÁCTICOS}

\section{Diseño del material didáctico:}

* Determinación de objetivos de aprendizaje.

* Especificación de criterios de destreza.

* Selección de medios y métodos.

* Adaptación de la enseñanza.

Determinación de elementos para la Conducción de la Asesonía:

a.- Establecimiento de reglas.

b.- O rientación del aprendizaje del estudiante.

c.- Conducción de la enseñanza.

- Buena Organización del curso.

- Rutinas claras

- Manejo apropiado del comportamiento

\section{Procedimientos de enseñanza:}

a.- Establecimiento del propósito que busca lograr el curso.

b.- Presentación de la información.

c.- Corrección de errores.

d.- D eterminación de actividades de aprendizaje.

e.- Verificación de cargas de trabajo.

\section{Proceso de evaluación:}

a.- Autoevaluación.

b.- Verificación formativa.

\section{Diseño del material didáctico:}

* Determinación de objetivos de aprendizaje.

D eben establecerse objetivos relevantes y apropiados para inducir el aprendizaje. Los objetivos deben establecer claramente el propósito del curso.

* Especificación de criterios de destreza.

D eben establecerse los procedimientos para que el estudiante pueda observar el progreso en su aprendizaje y el asesor pueda verificarlo. Habrá que determinar cómo pueden demostrar los estudiantes su dominio de las metas y objetivos de aprendizaje.

* Selección de medios y métodos.

Se asigna un valor didáctico al uso de un medio en particular, v.g., la utilización del audiocasette para proporcionarle al estudiante información para contextualizar un tema. 
Las variables de asesoría se tienen en cuenta en la planificación del material. Se asigna el tiempo suficiente para las actividades de aprendizaje, cuidando que éstas se relacionen claramente con los objetivos de aprendizaje.

\section{* Adaptación de la enseñanza.}

Los materiales deben especificar claramente los conocimientos previos que necesita el estudiante para utilizar con eficacia el material. El asesor debe sugerir lecturas o elaboración de materiales complementarios para que los estudiantes los adapten a grupos específicos.

La asignación de actividades de aprendizaje y autoevaluación debe permitir que los estudiantes desarrollen sus propios intereses a partir de los conocimientos previos adquiridos. El nivel adquirido deberá permitirles expresar los objetivos del aprendizaje, sin caer en contradicciones ni abusar de la generalización, el reduccionismo, o la repetición tediosa. Las actividades de aprendizaje y autoevaluación deben establecer claramente su sentido y finalidad.

\section{Determinación de elementos para conducir la asesonía: \\ * Establecimiento de reglas}

Establecer reglas claras y relevantes que rijan las relaciones entre asesory estudiante para que ambos sepan cómo se espera que se comporten.

* Orientación del aprendizaje del estudiante.

Enfatizar el valor del autoaprendizaje, determinar medios para que el estudiante pueda conducir su propio aprendizaje y señalar los posibles errores que se detecten en los materiales didácticos. Sugerir actividades que puede realizar el asesor para reforzar una conducta orientada al aprendizaje.

* Conducción de la enseñanza.

Señalar los elementos que deben considerarse para lograr una conducción eficiente del curso, e indicar los posibles problemas que puedan presentarse en los siguientes aspectos:

- Organización del curso.

- Rutinas a cumplir por parte de estudiantes y asesores.

- Comportamiento esperado del estudiante.

* Procedimientos de enseñanza:

Propósito a cumplir con el curso: establecer explícitamente el propósito del curso; dar un panorama sobre el material, la información incluida y el uso de los materiales; comunicar las expectativas que se espera cumplir, y señalar la importancia de los conocimientos adquiridos.

Presentación de la información. Verificar que las orientaciones de aprendizaje sean claras y comprensibles. Presentar de un modo explícito y organizado las habilidades que se pretende desarrollar en el alumno, o los conceptos que debe comprender y manejar vinculándolos a amplias oportunidades de práctica de aprendizaje guiada. 
Corrección de errores. Utilizar las actividades deaprendizajey autoevaluación de los estudiantes como instrumento para corregir errores, y ajustar los procedimientos de aprendizaje conforme a las necesidades de los alumnos.

D eterminación de actividades de aprendizaje. Las actividades de aprendizaje deben asignar trabajo suficiente, pertinente y variado, adecuado a las habilidades que debe desarrollar el estudiante. Verificación de cargas de trabajo.

\section{* Proceso de evaluación:}

Las actividades de aprendizaje y autoevaluación deben conducir al alumno a reflexionar sobre el proceso de aprendizaje. D eben inducir al alumno a observar desde dentro el proceso de aprendizaje: ¿Q ué estrategia fue más útil? ¿Cómo comprendió mejor un tema, haciendo uso de un mapa semántico 0 por medio de ejercicios de lectura de comprensión?

Mostrar al estudiante cómo se da el aprendizaje sugiriéndole actividades que le den oportunidad de pensar, como por ejemplo escribir sobre los enfoques del aprendizaje. Inducir en el estudiante el valor de la escritura como medio de comunicación.

\section{* Venificación formativa:}

Establecer medios para que los estudiantes y asesores puedan verificar los logros de aprendizaje una vez concluido un segmento del curso.

\section{EL ASE SOR}

La presencia del asesor es una parte esencial de las formas y procedimientos de la educación abierta, y abarca una amplia gama de actividades que van desde la aclaración de dudas derivadas de defectos en la construcción de los materiales didácticos o deficiencias en la formación del estudiante, hasta la función primordial de generar en el estudiante nuevas perspectivas y dudas acerca de lo aprendido.

Los asesores juegan un papel primordial al recoger la información necesaria para evaluar objetivamente la eficacia del sistema de enseñanza con objeto de retroalimentar racionalmente las necesarias adecuaciones, modificaciones o correcciones del material educativo.

El sentido semántico del asesor está vinculado a la idea de aconsejar, es decir sugerir, insinuar, influir en el ánimo de alguien, por tanto la conceptualización del asesor forma parte nodal del diseño de un modelo de educación a distancia debido a que su función no radica tanto en mostrar o demostrar los contenidos temáticos, acción que recae en los materiales didácticos, como en su capacidad para sugerir al estudiante alternativas en el desarrollo de su proceso de enseñanza-aprendizaje.

Un asesor no puede, en sentido estricto, preconfigurar una idea exacta de la manera en la que habrá de desarrollarse su función de asesoría, ésta quizás sea una diferencia esencial con el maestro en el aula. Puesto que no es factible que el asesor se imagine con certeza el tipo de circunstancias que puede enfrentar en el desarrollo de 
sus funciones (aunque existen elementos que se repiten), siempre estará sujeto al ensayo de prueba y error para enfrentar el desarrollo de un curso.

Los métodos y técnicas conocidas sirven como punto de partida para controlar las circunstancias en las que puede encontrarse en un momento dado, y también ser aprovechados para su formación profesional. Empero, la observación sistemática de fenómenos que se repiten, así como la reflexión sobre las formas de solución y respuesta, y su relación con los resultados que se obtienen, son caminos que pueden conducir al aprendizaje de las diversas formas que puede adoptar la asesoría.

\section{Funciones del asesor}

1.- Función orientadora:

Orientar al alumno en sus estudios, sobre todo en métodos de estudio:

Lectura comprensiva de los textos de estudio

Lectura de la bibliografía complementaria

Búsqueda de fuentes bibliográficas

Estudio de problemas vinculados con actividades prácticas

Preparación de trabajos

Preparación de exámenes

\section{La función orientadora debe:}

D etectar las necesidades de los alumnos en relación a sus aprendizajes por medio de la revisión y el seguimiento de sus trabajos.

Ayudar al alumno a tomar conciencia de las características de su aprendizaje. A poyar al alumno a adoptar una posición de aprendizaje activo que lo lleve a una búsqueda continua de preguntas y al planteamiento de problemas, más que fomentar en él una disposición a solucionar problemas y responder preguntas.

\section{Función del asesor en la evaluación de la enseñanza:}

El profesor tutor es intermediario entre el profesor del curso y los alumnos, y por tanto está en una situación privilegiada para identificar las principales áreas de dificultad que enfrenten los materiales didácticos.

La relación asesor alumno se prevé basada en los siguientes aspectos: a):- Inducción al sistema de autoestudio.

El objetivo es crear material didáctico que induzca al estudiante al estudio y la evaluación por medio del sistema a distancia. La función del asesor será la de motivary reforzar las actitudes del alumno hacia el autodidactismo, valiéndose de los ejercicios que el alumno debe resolver.

b):-Reforzamiento de actitudes y procesos de autoestudio.

Lafunción del asesor implicará dar seguimiento a las tareas deautoaprendizaje del alumno, orientar y controlar los resultados que obtenga, y sugerir actividades que tiendan a desarrollar las habilidades didácticas de los alumnos. 
Se prevé que la evaluación se integre en el proceso de aprendizaje y sea continua y le proporcione al alumno una orientación individualizada a lo largo del proceso de aprendizaje. La evaluación será de dos tipos:

- Ejercicios de autoevaluación al final de cada unidad didáctica.

- Pruebas de evaluación a distancia; su finalidad seríala de ayudar al estudianteair sistematizando su propio aprendizaje y sentirse orientado y motivado al comprobar su aprovechamiento.

\section{Promoversituaciones que le permitan al alumno confrontar sus conocimien- tos y opiniones con las de otros.}

La asesoría adoptará la forma de foros y listas en las que la discusión de un tema estaráa cargo de varios especialistas y donde existirála posibilidad de que el alumno participe por medio de preguntas o con opiniones propias.

En la comunicación educativa el uso de cualquier tipo de técnica o situación no garantiza absolutamente nada, puesto que el proceso de comunicación depende de diversos factores ligados a cuestiones de índole subjetiva (interés por decir, 0 escuchar), estados de ánimo, condiciones ambientales. La comunicación educativa está sujeta a multitud de variables, algunas de las cuales son controlables; no obstante es factible presuponer que ciertas técnicas y condiciones favorecen la comunicación. El uso del correo electrónico y las listas electrónicas, permite crear un ambientedepetenenia a una comnidado un ambiente digital de comunicación en múltiples direcciones: alumno-institución, alumno-alumno, alumno-asesor, además de que puede haber varios tipos de participación: enviando-recibiendo mensajes, o bien simplemente observando los mensajes.

Las actividades de asesoría deben planearse a partir de considerar elementos como los siguientes:

1. Claridad en la presentación de los mensajes didácticos o de información necesarios para el desarrollo del curso.

2. Procesos de retroalimentación como mecanismos de control útiles para la evaluación de la eficacia del proceso de enseñanza aprendizaje.

3. Medios de interacción a través de seminarios virtuales programados, vídeo conferencias, y encuentros de estudiantes.

4. Uso de medios de comunicación interactiva, como el chat.

5. Utilización de medios auditivos y visuales, no sólo para transmitir mensajes didácticos sino también para proporcionar elementos que induzcan la comunicación entre los sujetos participantes en el proceso de aprendizaje.

6. Servicios de información bibliográfica y noticiosos y de seguimiento del calendario del curso. 


\section{EN CONCLUSIÓN}

Un modelo de comunicación educativa a distancia debe valerse de todos los recursos a su alcance para generar un entorno de comunicación que establezca la idea de una comunidad deaprendizaje, aunqueésta no exista de facto en un tiempo-espacio físico determinado.

El aumento en la generación de posibilidades tecnológicas y de comunicación que pueden ser aplicadas a la educación a distancia indica que esta modalidad será una de las preferidas para incidir en las convergencias tecnológicas que se aplican en los procesos educativos. Una de ellas se relaciona con Internet 2, que tiene como uno de sus propósitos incidir en la educación superior, así como incrementar portales cuyo contenido puede ser general o especializado, local o internacional y contar con diversos servicios de comunicación remota. Es éste un indicio importante de que internet afectará, de alguna manera, diversos procesos educativos.

Asimismo se percibe una constante demanda, por parte de egresados de escuelas de Bibliotecología, por propuestas educativas que les permitan, entre otros aspectos, actualizar sus conocimientos o elevar sus niveles académicos, lo cual podría realizarse mediante programas a distancia bien estructurados y con la participación de especialistas del área y de instituciones interesadas en incursionar en esta modalidad educativa.

\section{BIBLIOGRAFÍA CONSULTADA}

Bear, John y Mariah Bear. Cdleegedegees bymail: and modm100 areeited shods that offer badhdor's master, dottartes, andLawdegresbyhomestudy. Berkeley, California : Ten Speed Press, 1999. 203p.

Casas A rmegol, Miguel. Univesidddsindaese edraáónadistaniaenAméicaLa tima Caracas : Kapeluz, OEA-UNA, 1987, 220p.

Colom Cañellas, Antonio J. Pedagogíatecnológica para la educación a distancia. En Losnuabsescenariosedrativosylastransfomadionstemnógicas Compiladora Patricia Ávila Muñoz. México : ILCE, 1998. pp.25-40

Cruz Rincón, Jaime. Citado por Casas Armegol, Miguel. En Univesidadsinda ses: eduracióna distania enA méica Latina. Caracas : O EA, Coordinación de Universidad Abierta y a Distancia, 1986. 341p.

Chan, María Elena, Adriana Villaseñory otros. "Guía para la elaboración del paquete de materiales didácticos orientados al aprendizaje independiente". En EstudioIndqpendiente México : ILCE, 1996. pp. 259-282.

D íaz Barriga, Frida. "El aprendizaje significativo desde una perspectiva constructivista." ED UCAR. 1993: Citada por G uillermo G arcía O lvera. En Propuesta para elaborar programas de actualización a distancia para egresados de escuelas mexicanas de bibliotecología (tesis de Maestría, en proceso) 
Floridi, Luciano. "The Internet: which future for organized knowledge, Frankenstein or Pygmalion? part. 1". EletronicLibrary. 14:1. February 1996. pp. 56.

Holemberg, Borje. Educacóna distanaia: situacónypespetivas Buenos Aires : Kapeluz, 1985. 180p.

- - - . Citado por Casas Armegol, Miguel. En Univesidadsindases eduracón a distancia enA Améica Latima, 1981, 341p.

- - - . Kaye y Casas. Citados por Stojanovic, Lily . "Teorías en la educación universitaria a distancia de América Latina: exploración sobre la utilización de bases teóricas en la producción de materiales instruccionales escritos". En Revistadeteendoǵa Edur cativa, 10(4), 1988, pp. 273-291.

Information Management Report: an international newsletter for information professionals and librarians. England: Elsevier Science, 1996. 19p.

Jones Shoemaker, Cynthia C. Leadeship in contining and distance eeducation in higher eduration Boston : Allyn and Bacon, 1998. 267p.

Keegan, D esmond. Foundations of distance education, 3th edition. London : Routledge, 1996. 224p.

- - - . "A theory for distance education." En Contempararyissuesin American distanceeduration Oxford : Pergamon Press, 1990. pp. 327-331.

Lafuente López, Ramiro. Elaboracóndetextosparaenseñarbiblideeo loǵa[D ocumento en proceso, 1999]

Libraries and dher acadenic support services for distanceleaming edited by Carolyn A. Snyder and James W. Fox. G reenwich, Connecticut : Jai Press Inc., 1997. 334p.

Main, Linda. Web-based virtual classrooms : a model for LIS education. En Edurationfor Information 16(4), december 1998. pp. 333-340.

Merril, M. D. Citado por Stojanovic, Lily. "Teorías en la educación universitaria a distancia de América Latina: exploración sobre la utilización de bases teóricas en la producción de materiales instruccionales escritos". En Reista detendoǵaEducativa, 10(4), 1988, pp. 273-290.

Losnuevosescenariosedurativosylastransformacionestemdógicas / Compiladora Patricia Ávila Muñoz. México : ILCE, 1998. 79p. 
Peña Borrero, Luis Alberto. Planeacóndeprogramas deuniversidadabietayadis tanaia: factorescúticos parala toma dedeisiones Colombia : Ministerio de Educación Nacional, 1983. 66p.

Piters. Citado por Stojanovic, Lily . "Teorías en la educación universitaria a distancia de A mérica Latina: exploración sobre la utilización de bases teóricas en la producción de materiales instruccionales escritos". En Reistade teendoǵa Educativa, 10(4), 1988, pp. 273-290.

Rumble y Harry. Citados por Casas Armegol, Miguel. En Universidadsindases educadón a distania enAméica Latina Caracas : Kapeluz, OEA-UNA, 1986, 220p.

Rumble, Genville. "On defining distance education". En Antdoǵadd] Diplo mada usosdelasnuevasteendoǵasenlaeduracoón México : UNAM, CISE, 1995. PP.63-77

Sánchez, V. Saadiay Estrella Pérez M. Rellexionessdbrelaformacóndereursoshur manos de cuarto nived en e área de las demias dela infomadón En INFO LAC. 9(4),1996.

Stojanovic, Lily. "Teoría en la educación universitaria a distancia de América Latina: exploración sobre la utilización de bases teóricas en la producción de materiales de instrucción". En Revista deTendoǵa Educativa, 10(4), pp 273-290, 1988.

Unwin, Lorna, Kate Stephens y Neil Bolton. Therdeofthelibraryindistancelearning London : Bowker, 1998. 256p.
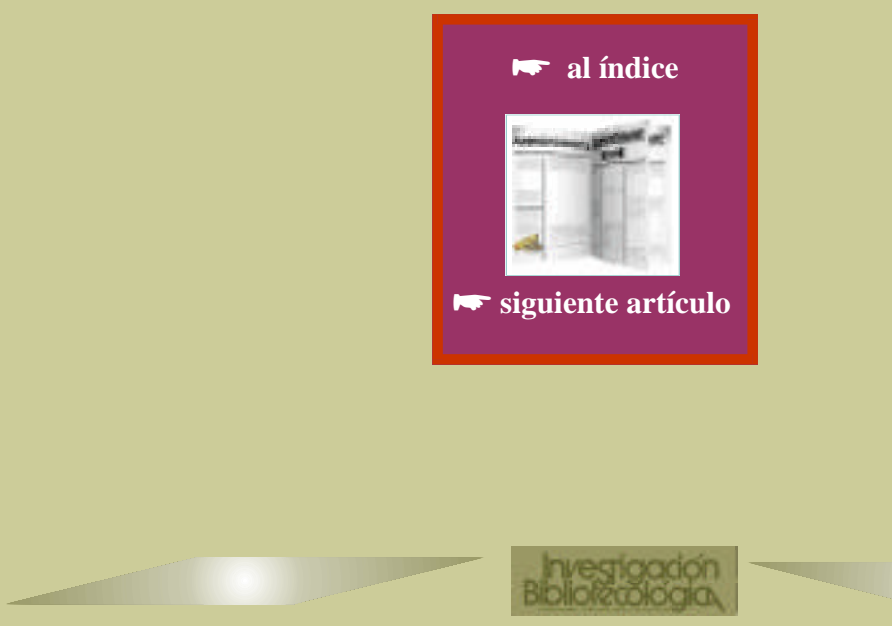